\title{
INDICADORES DE BEM-ESTAR BASEADOS EM REAÇÕES COMPORTAMENTAIS DE MATRIZES PESADAS
}

\author{
DANILO F. PEREIRA ${ }^{1}$, IRENILZA DE A. NÄÄS ${ }^{2}$, CARLOS E. B. ROMANINI ${ }^{3}$, \\ DOUGLAS D. SALGADO ${ }^{4}$, GRACELY O. T. PEREIRA ${ }^{5}$
}

\begin{abstract}
RESUMO: Dada a importância da avicultura de corte para a economia brasileira, este trabalho buscou compreender e identificar novos indicadores de bem-estar inerentes ao animal que contribuíssem para o aumento da eficácia produtiva, estudando-se diferentes reações comportamentais de matrizes de frango de

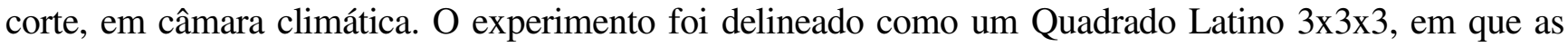
variáveis temperatura do ar, ração e idade das aves foram controladas, sendo as aves de diferentes idades alojadas em boxes distintos. Foram feitas observações do comportamento das aves em dois horários do dia, sendo uma observação na parte da manhã e outra na parte da tarde, durante períodos de 15 minutos cada, por meio de câmeras de vídeo instaladas no teto da câmara climática, não havendo interferência humana no registro das informações. Constatou-se, nos dados, a influência das variáveis controladas em diversos comportamentos observados, concluindo-se que a presença do alimento é um fator experimental relevante, pois aumenta as reações comportamentais agressivas.
\end{abstract}

PALAVRAS-CHAVE: avicultura, imagens de vídeo, bem-estar animal.

\section{WELFARE POINTERS IN FUNCTION OF BEHAVIOR REACTIONS OF BROILER BREEDERS}

\begin{abstract}
Knowing the importance that the poultry industry represents for the Brazilian economy, this work, searched to understand and to identify new welfare pointers inherent to the animal that contributed for the increase of the productive effectiveness, studying different behavior reactions in broiler breeders, in climatic chamber. The experiment was delineated as a Latin Square 3x3 3 , where the variable: temperature of air, birds ration and birds age had been controlled. The birds of different ages had been lodged in distinct boxes. Observations of the behavior of the birds in two schedules of the day had been made, being one in the morning and the other one in the afternoon, during a period of 15 minutes each through video cameras, installed in the ceiling of the climatic chamber, having no interference of human being in the register of the data. It was verified the influence of the controlled variables in diverse observed behaviors where it was concluded that the presence of food resulted in bigger occurrences of aggressiveness reactions.
\end{abstract}

KEYWORDS: poultry, video images, animal welfare.

\footnotetext{
${ }^{1}$ Eng ${ }^{0}$ Agrícola, Prof. Assistente, UNESP, UDT-Tupã - SP, Fone: (0XX14) 3404.4200, danilo@ tupa.unesp.br

${ }^{2}$ Prof. Titular, FEAGRI-UNICAMP, Campinas - SP, Fone (0XX19) 3788.1039, irenilza@ agr.unicamp.br

${ }^{3}$ Estudante de Graduação, FEAGRI-UNICAMP, Campinas - SP, romanini@ agr.unicamp.br

${ }^{4}$ Mestrando, FEAGRI-UNICAMP, Campinas- SP.

${ }^{5}$ Graduanda em Engenharia Ambiental, PUC, Campinas - SP.

Recebido pelo Conselho Editorial em: 8-9-2004

Aprovado pelo Conselho Editorial em: 24-6-2005
} 


\section{INTRODUÇÃO}

Atualmente, a avicultura nacional está passando por um processo de modernização com altos investimentos em ferramentas tecnológicas para mensuração e controle da produção e que permitam redução de perdas e maximização de receitas. Esse processo está sendo fundamental para a garantia da competitividade do frango brasileiro e conquista de novos mercados. Porém, apesar de toda essa modernização, o controle da produção avícola não está sendo o mais eficaz, uma vez que as variáveis medidas, que são responsáveis pelo acionamento e controle de sistemas automáticos, são indiretas, e não representam efetivamente o bem-estar ou necessidade dos animais. $\mathrm{O}$ desafio é, portanto, o desenvolvimento de metodologias para avaliação das reais necessidades ambientais das aves e que possam ser utilizadas para acionamento de controles de sistemas de climatização e alimentação, de forma que possam garantir maior eficácia na produção.

Ao encontro das demandas de mercado, diversos autores vêm pesquisando o bem-estar animal utilizando tecnologias complexas, devido à importância deste tema na atualidade (MARÍA et al. 2004; AL-AWADI et al., 1995; MARCHANT et al., 2001; PEREIRA, 2003). Como as variáveis fisiológicas são difíceis de medir em condições de campo, os estudos do comportamento têm se mostrado os mais viáveis para inferir sobre os níveis de bem-estar para aves alojadas. $\mathrm{O}$ animal é fortemente influenciado no seu comportamento pelo ambiente externo, e conhecendo como esse atua sobre o animal por meio do comportamento, é possível identificar e quantificar o bem-estar dos animas. GRAVES (1982) conceitua o comportamento animal como sendo uma janela entre o organismo vivo e o exterior, ou seja, o ambiente externo, que é composto pelas variáveis climáticas e sociais, atua sobre o animal positiva ou negativamente, e esse reage, dentre outros mecanismos biológicos, morfológicos e fisiológicos, através do seu comportamento.

Os aspectos sociais, principalmente os baseados no comportamento das aves, tornam-se cada vez mais evidentes na exploração avícola moderna, face à importância do ambiente em que as aves estão sujeitas. CAMPOS (2000) considera fundamental a identificação de fatores responsáveis pelo bemestar das aves, uma vez que a exploração avícola atual, fundamentalmente, é baseada na mudança de comportamento das aves. Há que se destacar, também, o relato de SNOWDON (1999) sobre o desenvolvimento alcançado em importantes conceitos relativos à adaptação ao estresse, em função das pesquisas com animais.

Diante desse contexto, o presente trabalho estudou o comportamento de matrizes pesadas de frango de corte, em câmara climática, aplicando-se três níveis de temperatura do ar, mediante três níveis de idade das aves e três formulações diferentes de ração. O objetivo foi demonstrar a viabilidade da utilização de equipamentos de precisão, como, por exemplo, o monitoramento eletrônico dos animais por câmeras de vídeo, na identificação de diferentes respostas comportamentais e possível identificação de comportamentos que sirvam como indicadores do bem-estar das aves em função dos fatores experimentais controlados. $\mathrm{O}$ objetivo deste trabalho não se limitou a provar com exatidão os resultados dos comportamentos que são afetados pelos fatores experimentais, mas, sim, apontar de maneira científica e intuitiva potenciais indicadores de bem-estar baseados em comportamentos de rotina de matrizes pesadas alojadas.

\section{MATERIAL E MÉTODOS}

O experimento foi conduzido no interior de uma câmara climática, com controle eletrônico de temperatura e umidade do ar, no Laboratório de Conforto Térmico II, da Faculdade de Engenharia Agrícola (FEAGRI/UNICAMP), em Campinas - SP. Utilizou-se, no estudo, um total de 27 matrizes pesadas da linhagem Hybro-PG, divididas em três lotes de idades, sendo: Id1 = 38 semanas; Id $2=46$ 48 semanas, e Id3 = 56 semanas. Dentro da câmara climática, as aves foram alojadas separadamente 
em três boxes, garantindo, dessa forma, a mesma condição ambiental para os três grupos de aves com diferentes idades (Figura 1).
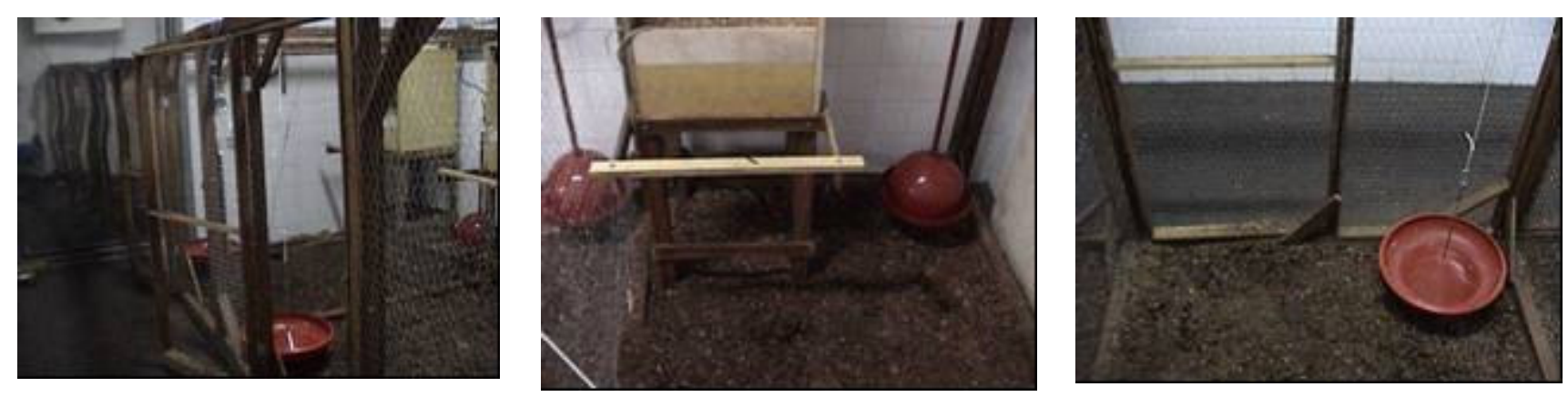

FIGURA 1. Imagem dos boxes montados dentro da câmara climática.

Das condições ambientais, a umidade relativa foi fixada em $75 \%$ e esta, efetivamente, variou entre $70 \%$ e $85 \%$, considerado por TINÔCO (2001) como permissível, ou dentro da zona de termoneutralidade. Variou-se a temperatura do ar em três níveis, sendo eles: $\mathrm{T} 1=13{ }^{\circ} \mathrm{C} \pm 2{ }^{\circ} \mathrm{C}$; $\mathrm{T} 2=$ $21^{\circ} \mathrm{C} \pm 2{ }^{\circ} \mathrm{C}$, e $\mathrm{T} 3=35^{\circ} \mathrm{C} \pm 2{ }^{\circ} \mathrm{C}$. A escolha dessas temperaturas baseou-se, entre outros, na afirmação de COSTA (1994), de que a temperatura ótima de conforto de matrizes pesadas é em torno de $21^{\circ} \mathrm{C}$. Foram fornecidas, ainda, três composições energéticas diferenciadas de ração para as aves $(\mathrm{R} 1=$ $2.810,81 \mathrm{kcal} \mathrm{kg}^{-1}$; R2 = 2.800,01 kcal kg${ }^{-1}$ e R3 = 2.789,71 kcal kg-1). Como ferramenta eletrônica de precisão, foram utilizadas três minicâmeras de vídeo coloridas que foram instaladas no teto da câmara climática, a três metros de altura, com visão de topo das aves, sobre cada um dos boxes das aves, com a função de registrar, por meio de vídeo digital, todas as reações comportamentais das matrizes, em seus respectivos lotes, em tempo real, conforme mostra a Figura 2.
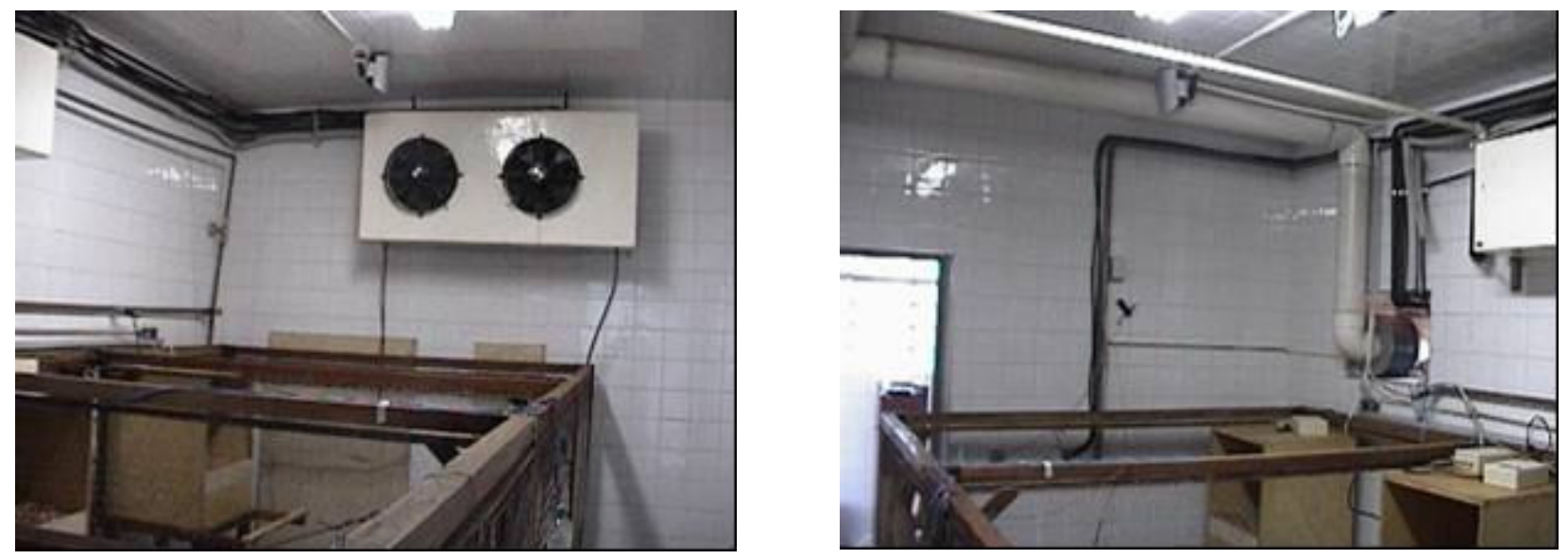

FIGURA 2. Câmeras de vídeo instaladas no teto da câmara climática.

O estudo foi delineado como um Quadrado Latino 3x3x3 (três níveis de temperatura, de idade e de ração). Esse método consiste em analisar cada variável comportamental em função dos níveis dos fatores predeterminados, assim como ilustrado na Tabela 1. O presente delineamento experimental estatístico foi adotado a fim de otimizar a logística experimental, permitindo redução no tempo de coleta dos dados e no número de aves (um terço do número de aves necessárias para a realização de um experimento fatorial). 
TABELA 1. Distribuição dos níveis dos fatores no método de quadrado latino.

\begin{tabular}{cccc}
\hline Lotes & Dias $1 ; 2$ e 3 & Dias $4 ; 5$ e 6 & Dias $7 ; 8$ e 9 \\
& T1 & T2 & T3 \\
\hline Id1 & R1 & R3 & R2 \\
Id2 & R2 & R1 & R3 \\
Id3 & R3 & R2 & R1 \\
\hline
\end{tabular}

As observações dos comportamentos foram feitas em dois períodos (matutino, compreendido entre os horários das 7 às 12 h, e vespertino, compreendido entre os horários das 12 às 19 h), devido ao fato de esses diferentes períodos trazerem possíveis mudanças comportamentais identificáveis. $\mathrm{O}$ tempo de coleta de dados para cada combinação de níveis de fatores mostrados na Tabela 1 foi de três dias e, para cada combinação, utilizaram-se três matrizes pesadas. A metodologia da coleta de dados do comportamento animal utilizada foi descrita por BIZERAY et al. (2002), permitindo a verificação do número de ocorrências de determinada reação comportamental por um período contínuo de observação de 15 minutos pela manhã e 15 minutos à tarde. As variáveis referentes às reações comportamentais observadas foram as mesmas em ambos os períodos do dia, sejam elas: limpar penas, espojamento, espreguiçar, prostrar, correr, deitar, ciscar, abrir asas, arrepiar penas, ameaçar, perseguir, montar e bicar, sendo as quatro últimas denominadas de reações comportamentais agressivas.

A partir da coleta e análise dos dados, buscou-se identificar, por meio de comparações múltiplas estatísticas, comportamentos que sofreram mudanças significativas devido aos fatores experimentais aplicados. Dessa forma, pretendeu-se identificar comportamentos que são potenciais indicadores de bem-estar animal, ou seja, os comportamentos que são mais afetados devido às variações do ambiente oferecido.

\section{RESULTADOS E DISCUSSÃO}

Os dados foram analisados por meio do teste de comparações múltiplas de Tukey, respeitando-se os períodos de coleta (matutino e vespertino), e divididos em termos de reações comportamentais ditas normais e agressivas, conforme mostram as tabelas abaixo. Devido à influência da presença de ração no período da manhã, que afetou os comportamentos nesse período, nesta análise as discussões foram agrupadas em função do período de registros.

TABELA 2. Média de ocorrência das reações comportamentais observadas no período da manhã $(\alpha=5 \%)$.

\begin{tabular}{|c|c|c|c|c|c|c|c|c|c|}
\hline & $\begin{array}{l}\text { Limpar } \\
\text { Penas }\end{array}$ & $\begin{array}{l}\text { Espoja- } \\
\text { mento }\end{array}$ & $\begin{array}{l}\text { Espre- } \\
\text { guiçar }\end{array}$ & Prostrar & Correr & Deitar & Ciscar & Abrir Asas & $\begin{array}{c}\text { Arrepiar } \\
\text { Penas }\end{array}$ \\
\hline $\mathrm{T} 1$ & $0,78 \mathrm{a}$ & $0,00 \mathrm{a}$ & $0,00 \mathrm{~b}$ & $0,00 \mathrm{a}$ & $2,00 \mathrm{a}$ & $0,67 \mathrm{a}$ & $0,78 \mathrm{a}$ & $1,33 a$ & $1,78 \mathrm{a}$ \\
\hline $\mathrm{T} 2$ & $0,33 \mathrm{a}$ & $0,00 \mathrm{a}$ & $0,22 \mathrm{ab}$ & $0,00 \mathrm{a}$ & $0,22 \mathrm{a}$ & $0,22 \mathrm{a}$ & $0,89 \mathrm{a}$ & $1,11 \mathrm{a}$ & $0,78 \mathrm{ab}$ \\
\hline $\mathrm{T} 3$ & $0,11 \mathrm{a}$ & $0,00 \mathrm{a}$ & $0,67 \mathrm{a}$ & $0,00 \mathrm{a}$ & $0,67 \mathrm{a}$ & $0,00 \mathrm{a}$ & $1,22 \mathrm{a}$ & $0,56 a$ & $0,33 b$ \\
\hline Id1 & $0,44 a$ & $0,00 \mathrm{a}$ & $0,56 a$ & $0,00 \mathrm{a}$ & $2,33 a$ & $0,22 \mathrm{a}$ & $1,11 \mathrm{a}$ & $0,44 a$ & $0,89 \mathrm{a}$ \\
\hline Id 2 & $0,33 \mathrm{a}$ & $0,00 \mathrm{a}$ & $0,22 \mathrm{a}$ & $0,00 \mathrm{a}$ & $0,22 b$ & $0,11 \mathrm{a}$ & $1,11 \mathrm{a}$ & $1,33 \mathrm{a}$ & $1,00 \mathrm{a}$ \\
\hline Id3 & $0,44 a$ & $0,00 \mathrm{a}$ & $0,11 \mathrm{a}$ & $0,00 \mathrm{a}$ & $0,33 b$ & $0,56 \mathrm{a}$ & $0,67 \mathrm{a}$ & $1,22 \mathrm{a}$ & $1,00 \mathrm{a}$ \\
\hline R1 & $0,44 a$ & $0,00 \mathrm{a}$ & $0,00 \mathrm{a}$ & $0,00 \mathrm{a}$ & $1,56 \mathrm{a}$ & $0,22 \mathrm{a}$ & $0,22 \mathrm{a}$ & $0,22 b$ & $0,56 \mathrm{a}$ \\
\hline $\mathrm{R} 2$ & $0,56 a$ & $0,00 \mathrm{a}$ & $0,56 a$ & $0,00 \mathrm{a}$ & $1,00 \mathrm{a}$ & $0,33 a$ & $1,22 \mathrm{a}$ & $2,00 \mathrm{a}$ & $1,44 \mathrm{a}$ \\
\hline R3 & $0,22 \mathrm{a}$ & $0,00 \mathrm{a}$ & $0,33 \mathrm{a}$ & $0,00 \mathrm{a}$ & $0,33 \mathrm{a}$ & $0,33 \mathrm{a}$ & $1,44 \mathrm{a}$ & $0,78 \mathrm{ab}$ & $0,89 \mathrm{a}$ \\
\hline
\end{tabular}

Nota-se, na Tabela 2, que os animais que estiveram submetidos à condição térmica $\mathrm{T} 3$ ( $\mathrm{T}=$ $35^{\circ} \mathrm{C}$ e UR $=75 \%$ ), são os que apresentam maior frequiência de espreguiçar, e as aves que foram estudadas sob a condição térmica $\mathrm{T} 1\left(\mathrm{~T}=13{ }^{\circ} \mathrm{C}\right.$ e UR $\left.=75 \%\right)$, arrepiam mais as penas. As aves mais 
jovens (Id1 = 38 semanas) foram as que mais apresentaram o comportamento de correr. As matrizes que se alimentaram da R2 $\left(2.800,01 \mathrm{kcal} \mathrm{kg}^{-1}\right)$ apresentaram freqüência maior de abertura das asas. Dado o controle dos fatores experimentais aplicados, esses comportamentos são potenciais indicadores do bem-estar dessas aves em função do fator ambiental atuante. De acordo com os conceitos de GRAVES (1982), esses comportamentos são reflexos diretos do ambiente sobre a ave, de modo que, conhecendo melhor como esses comportamentos são afetados por esses fatores isoladamente, é possível que se obtenham níveis de bem-estar que sejam função desses fatores.

TABELA 3. Ocorrência das reações comportamentais observadas no período da tarde $(\alpha=5 \%)$.

\begin{tabular}{cccccccccc}
\hline & $\begin{array}{c}\text { Limpar } \\
\text { Penas }\end{array}$ & $\begin{array}{c}\text { Espoja- } \\
\text { mento }\end{array}$ & $\begin{array}{c}\text { Espre- } \\
\text { guiçar }\end{array}$ & Prostrar & Correr & Deitar & Ciscar & $\begin{array}{c}\text { Abrir Asas } \\
\text { Arrepiar } \\
\text { Penas }\end{array}$ \\
\hline T1 & $6,11 \mathrm{a}$ & $0,00 \mathrm{a}$ & $0,33 \mathrm{a}$ & $0,00 \mathrm{~b}$ & $0,78 \mathrm{a}$ & $1,44 \mathrm{a}$ & $5,30 \mathrm{a}$ & $1,44 \mathrm{a}$ & $2,00 \mathrm{a}$ \\
T2 & $4,00 \mathrm{a}$ & $0,00 \mathrm{a}$ & $0,67 \mathrm{a}$ & $0,00 \mathrm{~b}$ & $0,22 \mathrm{a}$ & $2,00 \mathrm{a}$ & $1,70 \mathrm{~b}$ & $1,33 \mathrm{a}$ & $1,33 \mathrm{ab}$ \\
T3 & $3,40 \mathrm{a}$ & $0,00 \mathrm{a}$ & $0,78 \mathrm{a}$ & $3,67 \mathrm{a}$ & $0,33 \mathrm{a}$ & $0,00 \mathrm{~b}$ & $1,10 \mathrm{~b}$ & $0,67 \mathrm{a}$ & $0,11 \mathrm{~b}$ \\
\hline Id1 & $5,30 \mathrm{a}$ & $0,00 \mathrm{a}$ & $0,44 \mathrm{a}$ & $1,44 \mathrm{a}$ & $0,22 \mathrm{a}$ & $1,33 \mathrm{a}$ & $3,40 \mathrm{a}$ & $0,56 \mathrm{a}$ & $1,11 \mathrm{a}$ \\
Id2 & $4,90 \mathrm{a}$ & $0,00 \mathrm{a}$ & $1,22 \mathrm{a}$ & $1,00 \mathrm{a}$ & $0,89 \mathrm{a}$ & $1,22 \mathrm{a}$ & $2,70 \mathrm{a}$ & $1,56 \mathrm{a}$ & $1,22 \mathrm{a}$ \\
Id3 & $3,30 \mathrm{a}$ & $0,00 \mathrm{a}$ & $0,11 \mathrm{a}$ & $1,22 \mathrm{a}$ & $0,22 \mathrm{a}$ & $0,89 \mathrm{a}$ & $2,00 \mathrm{a}$ & $1,33 \mathrm{a}$ & $1,11 \mathrm{a}$ \\
\hline R1 & $5,20 \mathrm{a}$ & $0,00 \mathrm{a}$ & $1,00 \mathrm{a}$ & $1,22 \mathrm{a}$ & $0,22 \mathrm{a}$ & $1,56 \mathrm{a}$ & $3,40 \mathrm{a}$ & $1,00 \mathrm{a}$ & $1,33 \mathrm{a}$ \\
R2 & $4,30 \mathrm{a}$ & $0,00 \mathrm{a}$ & $0,00 \mathrm{a}$ & $1,44 \mathrm{a}$ & $0,78 \mathrm{a}$ & $0,78 \mathrm{a}$ & $2,90 \mathrm{a}$ & $1,67 \mathrm{a}$ & $0,67 \mathrm{a}$ \\
R3 & $4,00 \mathrm{a}$ & $0,00 \mathrm{a}$ & $0,78 \mathrm{a}$ & $1,00 \mathrm{a}$ & $0,33 \mathrm{a}$ & $1,11 \mathrm{a}$ & $1,80 \mathrm{a}$ & $0,78 \mathrm{a}$ & $1,44 \mathrm{a}$ \\
\hline
\end{tabular}

No período da tarde (pós-alimentação), é notória maior ocorrência de prostrações nas matrizes de frango que estiveram submetidas ao estresse térmico acima da zona de termoneutralidade $\left(\mathrm{T}=35^{\circ} \mathrm{C}\right.$ e $\mathrm{UR}=75 \%$ ) e à ocorrência do ato de deitar-se nas demais condições ambientais aplicadas no estudo. Houve variações significativas nos comportamentos de ciscar e arrepiar penas nas aves que estiveram submetidas à condição térmica T1. Por meio dessa análise, é possível identificar potenciais indicadores de bem-estar. Nesse caso, os fatores ração e idade não influenciaram nos comportamentos das aves, provavelmente devido ao fato de não haver mais a presença do alimento como fator atuante. Assim, a presença ou não de ração no ambiente em que a ave está inserida, constitui fator experimental atuante.

A seguir, são mostradas as reações agressivas das aves em função dos fatores experimentais.

TABELA 4. Reações comportamentais agressivas observadas no período da manhã ( $\alpha=5 \%)$.

\begin{tabular}{ccccc}
\hline & Ameaças & Perseguição & Monta & Bicada \\
\hline T1 & $0,67 \mathrm{a}$ & $1,11 \mathrm{a}$ & $0,11 \mathrm{a}$ & $5,20 \mathrm{a}$ \\
T2 & $0,00 \mathrm{a}$ & $0,11 \mathrm{~b}$ & $0,00 \mathrm{a}$ & $0,30 \mathrm{~b}$ \\
T3 & $0,00 \mathrm{a}$ & $0,00 \mathrm{~b}$ & $0,00 \mathrm{a}$ & $1,40 \mathrm{~b}$ \\
\hline Id1 & $0,11 \mathrm{a}$ & $1,00 \mathrm{a}$ & $0,11 \mathrm{a}$ & $3,90 \mathrm{a}$ \\
Id2 & $0,22 \mathrm{a}$ & $0,00 \mathrm{~b}$ & $0,00 \mathrm{a}$ & $0,90 \mathrm{a}$ \\
Id3 & $0,33 \mathrm{a}$ & $0,22 \mathrm{ab}$ & $0,00 \mathrm{a}$ & $2,20 \mathrm{a}$ \\
\hline R1 & $0,11 \mathrm{a}$ & $0,89 \mathrm{a}$ & $0,11 \mathrm{a}$ & $2,90 \mathrm{a}$ \\
R2 & $0,22 \mathrm{a}$ & $0,00 \mathrm{a}$ & $0,00 \mathrm{a}$ & $1,80 \mathrm{a}$ \\
R3 & $0,33 \mathrm{a}$ & $0,33 \mathrm{a}$ & $0,00 \mathrm{a}$ & $2,30 \mathrm{a}$ \\
\hline
\end{tabular}

De acordo com a Tabela 4, o fator temperatura afetou significativamente a frequiência de perseguições e bicadas efetuadas pelas aves, principalmente na condição térmica $\mathrm{T} 1\left(\mathrm{~T}=13^{\circ} \mathrm{C}\right.$ e UR $=$ $75 \%)$ abaixo da zona de conforto térmico das aves $(\mathrm{p}<0,05)$. Esse elevado número de comportamentos agressivos, quando comparado com o período da tarde (Tabela 5), reflete a acentuada disputa que ocorreu pela ração. Esse resultado aponta a necessidade de energia das aves para manter a 
temperatura corporal em ambiente agressivo pelo frio. A diferença de idade também afetou o nível de perseguição das aves, sendo as aves mais jovens as que mais praticaram as perseguições.

TABELA 5. Reações comportamentais agressivas observadas no período da tarde $(\alpha=5 \%)$.

\begin{tabular}{ccccc}
\hline & Ameaças & Perseguição & Monta & Bicada \\
\hline T1 & $0,00 \mathrm{a}$ & $0,00 \mathrm{a}$ & $0,00 \mathrm{a}$ & $0,11 \mathrm{a}$ \\
T2 & $0,00 \mathrm{a}$ & $0,00 \mathrm{a}$ & $0,00 \mathrm{a}$ & $0,00 \mathrm{a}$ \\
T3 & $0,00 \mathrm{a}$ & $0,00 \mathrm{a}$ & $0,00 \mathrm{a}$ & $0,00 \mathrm{a}$ \\
\hline Id1 & $0,00 \mathrm{a}$ & $0,00 \mathrm{a}$ & $0,00 \mathrm{a}$ & $0,00 \mathrm{a}$ \\
Id2 & $0,00 \mathrm{a}$ & $0,00 \mathrm{a}$ & $0,00 \mathrm{a}$ & $0,11 \mathrm{a}$ \\
Id3 & $0,00 \mathrm{a}$ & $0,00 \mathrm{a}$ & $0,00 \mathrm{a}$ & $0,00 \mathrm{a}$ \\
\hline R1 & $0,00 \mathrm{a}$ & $0,00 \mathrm{a}$ & $0,00 \mathrm{a}$ & $0,00 \mathrm{a}$ \\
R2 & $0,00 \mathrm{a}$ & $0,00 \mathrm{a}$ & $0,00 \mathrm{a}$ & $0,11 \mathrm{a}$ \\
R3 & $0,00 \mathrm{a}$ & $0,00 \mathrm{a}$ & $0,00 \mathrm{a}$ & $0,00 \mathrm{a}$ \\
\hline
\end{tabular}

De acordo com a Tabela 5, no período vespertino, correspondente ao período pós-alimentação, nenhum dos tratamentos impostos na câmara climática (temperatura, idade e ração) afetaram ou induziram o comportamento agressivo das aves. Esse resultado reforça a afirmação anterior de que a presença do alimento é um fator atuante no comportamento das aves alojadas e que o comportamento agressivo é decorrente da disputa, seja pelo alimento e pelo espaço, seja pela hierarquia e dominância sobre o grupo.

Resumindo os resultados encontrados acima, na Tabela 6, apresentam-se os comportamentos que foram afetados pelos fatores experimentais e que constituem os potencias indicadores de bem-estar das aves estudas neste trabalho.

TABELA 6. Resumo dos resultados obtidos.

\begin{tabular}{ccc}
\hline Variáveis Experimentais & Fatores que Afetam o Comportamento & Período \\
\hline Correr & Idade & Manhã \\
Abrir Asas & Ração & Manhã \\
Arrepiar Penas & Temperatura & Manhã e Tarde \\
Espreguiçar & Temperatura & Manhã \\
Prostrar & Temperatura & Tarde \\
Deitar & Temperatura & Tarde \\
Ciscar & Temperatura & Tarde \\
Bicadas & Temperatura & Manhã \\
Perseguição & Temperatura e Idade & Manhã \\
\hline
\end{tabular}

Obs: O período da manhã caracteriza o período de alimentação, e o período da tarde caracteriza o período pós-alimentar.

\section{CONCLUSÕES}

Dos resultados obtidos, foi possível, por meio da análise experimental e do uso das câmeras de vídeo como ferramenta de precisão, identificar potenciais indicadores de bem-estar das aves observadas em reações comportamentais, em função dos fatores experimentais controlados. Pode-se afirmar que a presença do alimento constituiu um fator experimental e, na determinação de comportamentos que indicam o bem-estar, esse deve ser obrigatoriamente considerado nas análises. Foi possível verificar a influência direta do ambiente térmico nos comportamentos das aves, demonstrando a viabilidade de buscar indicadores de bem-estar baseados no comportamento animal. 


\section{AGRADECIMENTOS}

À Nutron, à Granja São José e à Granja Ipê, pelo apoio desprendido, e à FAPESP e ao CNPq, pelo financiamento ao trabalho.

\section{REFERÊNCIAS}

AL-AWADI, A.A.; HUSSEINI, M.D.; DIAB, M.F.; AL-NASSER, A.Y. Productive performance of laying hens house in minimal shade floor pens and laying cages under ambient conditions in hot arid regions. Livestock Production Science, Amsterdam, v.41, n.3, p.263-9, 1995.

BIZERAY D.; ESTEVEZ I.; LETERRIER C.; FAURE F.M. Effects of increasing environmental complexity on the physical activity of broiler chickens. Applied Animal Behaviour Science, Vancouver, v.79, n.1, p.27-41, 2002.

CAMPOS, E.J. O comportamento das aves. Revista Brasileira de Ciência Avícola, Campinas, v.2, n.2, 2000, p.93-113.

COSTA, C.A. Pontos críticos do manejo de matrizes - manejo de matrizes. In: CONFERÊNCIA APINCO DE CIÊNCIA E TECNOLOGIA AVÍCOLA, 1994, Campinas. Anais... Campinas: Fundação Apinco de Ciência e Tecnologia Avícola, 1994. p.1-10.

GRAVES, H.B. Behavioral responses of poultry (chickens) to management systems. In: SYMPOSIUM OF MANAGEMENT OF FOOD PRODUCING ANIMALS, 1982, West Lafayette. Proceedings... Wes Lafayette: Purdue University, 1982. v.2, p.122-38.

MARCHANT, J.A.; ANDERSEN, H.J.; ONYANGO, C.M. Evaluation of an imaging sensor for detecting vegetation using different waveband combinations. In: COMPUTERS AND ELETRONICS IN AGRICULTURE, 2001, Foz do Iguaçu. Anais... Campinas, 2001. p.101-17.

MARÍA, G.A.; ESCÓS, J.; ALADOS, C.L. Complexity of behavioural sequences and their relation to stress coinditions in chickens (Gallus gallus domesticus): a non-invasive technique to evaluate animal welfare. Applied Animal Behavior Science, Vancouver, v.86, n.1, p.93-104, 2004.

PEREIRA, D.F. Avaliação do comportamento individual de matrizes pesadas (frango de corte) em função do ambiente e identificação da temperatura crítica máxima. 2003. 190 f. Dissertação (Mestrado em Construções Rurais e Ambiência) - Faculdade de Engenharia Agrícola, Universidade Estadual de Campinas, Campinas, 2003.

SNOWDON, C.T. O significado da pesquisa em comportamento animal. Estudo de Psicología, Natal, v.4, n.2, p.365-73, 1999.

TINÔCO, I.F.F. Ambiência e instalações na produção de matrizes avícolas. In: SILVA, I.J.O. (Ed.). Ambiência na produção de aves em clima tropical. Jaboticabal: SBEA, 2001. v.2, p.1-74. 